\title{
Inhalt
}

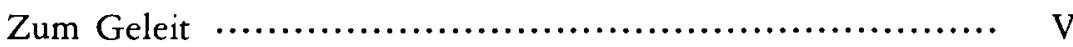

\section{Hans-Jürgen Ahrens}

Dr. jur., Professor an der Universität Osnabrück, Richter am Oberlandesgericht Celle

Einstweiliger Rechtsschutz als Hauptsacheverfahren im Wettbewerbsrecht

Rechtspolitische Überlegungen zum summarischen

Rechtsschutz ...........................................

Christian v. Bar

Dr. jur., Professor an der Universität Osnabrück, Direktor des Instituts für Internationales Privatrecht und Rechtsvergleichung, Bencher (Hons.) of Gray's Inn, London.

Die Haftung von Nebentätern, Teilnehmern und Beteiligten in den kontinentalen Deliktsrechten der Europäischen Union $\cdots 17$

GotTFried Baumgärtel

Dr. jur., Dr. jur. h. c. mult., em. Professor an der Universität zu Köln

Das Verhältnis von Beweislastumkehr und Umkehr der konkreten Beweisführungslast im deutschen Zivilprozeß $\ldots . . .41$

Kostas E. Beys

Dr. jur., o. Professor an der Universität Athen, Direktor des Studienzentrums zur Justizgewährung

Der Bereich der Privat- und Verwaltungsrechtsstreitigkeiten

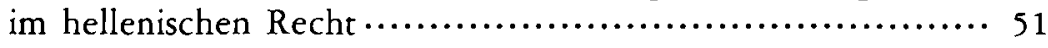

\section{Witold Broniewicz}

Dr. jur., o. Professor an der Universität Lódz

Die Berufung im polnischen Zivilprozessrecht 


\section{Dagmar Coester-W altjen}

Dr. jur., Professorin an der Universität München

Die Bedeutung des EuGVÜ und des Luganer Abkommens

für Drittstaaten

Hans W. Fasching

DDr. jur., em. o. Universitätsprofessor an der Universität Wien Rechtliches Gehör und Rationalisierung des zivilgerichtlichen Verfahrens in Österreich

\section{Hans Friedhelm Gaul}

Dr. jur., em. Professor an der Universität Bonn

Der Einfluß rückwirkender Gestaltungsurteile auf vorausgegangene Leistungsurteile

\section{Reinhold Geimer}

Prof. Dr. jur., Notar in München, Honorarprofessor an der Universität München „Internationalpädagogik" oder wirksamer Beklagtenschutz? Einige Bemerkungen zur internationalen Anerkennungszuständigkeit

\section{Peter Gottwald}

Dr. jur., Professor an der Universität Regensburg

Deutsche Probleme Internationaler Familienverfahren

\section{W Alther J. Habscheid}

Dr. jur., Dr. h. c. mult., em. o. Professor an der Universität Zürich, Professeur Honoraire de l'Université de Genève Zur materiellen Rechtskraft des Unzuständigkeitsentscheids

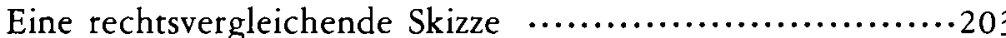

\section{Hans Hanisch}

Dr. jur., em. o. Professor der Rechte an der Universität Genf Grenzüberschreitende Insolvenz: Drei Lösungsmodelle im Vergleich 


\section{Andreas Heldrich}

Dr. jur., Professor an der Universität München, Direktor des Instituts für Internationales Recht - Rechtsvergleichung Probleme bei der Ermittlung ausländischen Rechts in der gerichtlichen Praxis

\section{Nikolaos K. KLamaris}

Dr. jur., o. Professor für Zivilprozeßrecht an der Universität Athen, Stellv. Direktor des Instituts für prozeßrechtliche Studien in Athen, Rechtsanwalt beim Areopag

Die Grundstruktur des griechischen Zwangsvollstreckungsrechts als des effektiven Teils des prozessualen Grundrechts auf Justizgewährung

Eine zusammenfassende Betrachtung

\section{HaRald Koch}

Dr. jur., Professor an der Universität Rostock, Richter am Oberlandesgericht Rostock

Rechtsvergleichende Fragen zum "Gesetzlichen Richter" .....281

\section{Dieter Leipold}

Dr. jur., Professor an der Universität Freiburg, Direktor des Instituts für Deutsches und Ausländisches Zivilprozeßrecht

Wahrheit und Beweis im Zivilproze $B . . . \ldots \ldots \ldots \ldots \ldots \ldots \ldots \ldots \ldots 1$

\section{W Alter F. Lindacher}

Dr. jur., Professor an der Universität Trier Internationale Zuständigkeit in Wettbewerbssachen

Der Gerichtsstand der Wettbewerbshandlung nach autonomem deutschem IZPR

\section{KARL-Georg LoRITZ}

Dr. jur., Professor an der Universität Mainz

Rechtsprobleme der Vertretung von Gesellschaften mit beschränkter Haftung im Zivilprozeß bei Unwirksamkeit von Anteilsübertragungen 


\section{KaZIMIERZ LUBiŃSKI}

Dr. jur., Professor an der Universität Toruń

Das Wesen und die juristische Natur der Tätigkeit des

Gerichts in der freiwilligen Gerichtsbarkeit

\section{Gerhard LüKe}

Dr. jur., Dr. h. C., em. Professor an der Universität des Saarlandes Die Entwicklung der öffentlichrechtlichen Theorie der Zwangsvollstreckung in Deutschland

\section{WoLFGaNG LüKE}

Dr. jur., Professor an der Technischen Universität Dresden Notarielles Vermittlungsverfahren nach dem Sachenrechtsbereinigungsgesetz Modell alternativer Streitbeilegung oder Regelung eines Sonderfalles?

\section{Hans-Joachim Musielak}

Dr. jur., Professor an der Universität Passau

Einige Gedanken zur materiellen Rechtskraft

\section{JánOS NÉmeth}

Dr. jur., Professor an der Eötvös Loránd Universität Budapest Zivilprozeß 3 und der Grundsatz der Öffentlichkeit in Ungarn 445

\section{Hanns Prütting}

Dr. jur., Professor an der Universität zu Köln, Direktor des Instituts für Verfahrensrecht

Grundsatzfragen des deutschen Rechtsmittelrechts

\section{W ALter H. RechBerger}

Dr. jur., o. Universitätsprofessor an der Universität Wien

Rechtssicherheit, Entscheidungsharmonie und Bindung an Vorfrageentscheidungen Überlegungen zu den objektiven Grenzen der Rechtskraft im österreichischen Zivilprozeßrecht 


\section{Haimo Schack}

Dr. jur., Professor an der Universität zu Kiel, Direktor des Instituts für Europäisches und Internationales Privat- und Verfahrensrecht

Internationale Zuständigkeit und Inlandsbeziehung

\section{Peter Schlosser}

Dr. jur., Professor an der Universität München

Wann ist ein Urteil aufgehoben?

Rolf A. Schütrze

Prof. Dr. jur., Rechtsanwalt in Stuttgart

Dissenting Opinions im Schiedsverfahren

\section{Daphne-Ariane Simotta}

Dr. jur., o. Universitätsprofessorin an der Karl-Franzens-Universität Graz

Die Entwicklung der Gerichtsbarkeit in Familiensachen in

Österreich

\section{Marcel Storme}

Dr. jur., Vorsitzender des interuniversitären Zentrums für Prozeßrecht (Belgien), President der internationalen Vereingung für Prozeßrecht

Ein einheitlicher Europäischer Vollstreckungstitel als

Vorbote eines weltweiten Titels

\section{ROLF STÜRner}

Dr. jur., Professor an der Universität Freiburg, Direktor des Instituts für Deutsches und Ausländisches Zivilprozeßrecht

Das französische und englische $Z$ wangsvollstreckungsrecht

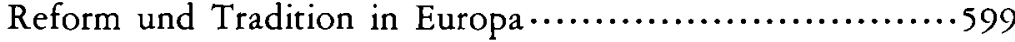

\section{Michele Taruffo}

Dr. jur., Full Professor of University of Pavia

Legal Cultures and Models of Civil Justice 


\section{Hans Ulrich W Alder}

Dr. jur., Rechtsanwalt, em. Professor an der Universität Zürich

Rechtsbehelfe im schweizerischen Bundesgesetz über

Schuldbetreibung und Konkurs

\section{Gerhard W Alter}

Dr. jur., Professor an der Universität Bern, Direktor des Instituts für Schweizerisches und Ausländisches Zivilprozessreche

Vorläufiger Rechtsschutz in der Schweiz

\section{Manfred Wolf}

Dr. jur., Professor an der Universität Frankfurt

Geständnis zu eigenen Lasten und zu Lasten Dritter?

\section{Pelayia Yessiou-Faltsi}

Dr. jur., The Dean of the School of Law and Economics Aristotle University of Thessaloniki

Judicial Review of Unconstitutional Legislation in Greece $\cdots 697$

\section{TAKeYoshi UchIDA}

Professor an der Waseda Universität

Hideo Nakamura

Seine wissenschaftliche Arbeit 